\title{
Superfluid Density and Angular Dependence of the Energy Gap in Optimally Doped $(\mathrm{BiPb})_{2}(\mathrm{SrLa})_{2} \mathrm{CuO}_{6+\delta}$
}

\author{
S. Strässle • R. Khasanov • T. Kondo • D.O.G. Heron • \\ A. Kaminski · H. Keller · S.L. Lee · T. Takeuchi
}

Received: 3 November 2008 / Accepted: 5 November 2008 / Published online: 25 November 2008

(C) Springer Science+Business Media, LLC 2008

\begin{abstract}
We present a muon-spin rotation study of the optimally doped cuprate superconductor $(\mathrm{BiPb})_{2}(\mathrm{SrLa})_{2}$ $\mathrm{CuO}_{6+\delta}$. The measured magnetic field dependence of the in-plane magnetic penetration $\lambda_{a b}$ suggests superconductivity with a dominant $d$-wave order parameter. The comparison of the temperature dependence of $\lambda_{a b}$ with calculations, assuming the angular gap symmetry as obtained from photoemission measurements, is consistent with a partial suppression of the quasi-particle weight towards the anti-nodal region of the Fermi surface. This suggests that the superconducting and the pseudogap state are dominated by different parts of the Fermi surface.
\end{abstract}

\section{S. Strässle $(\bowtie) \cdot$ H. Keller}

Physik-Institut der Universität Zürich, Universität Zürich, 8057 Zürich, Switzerland

e-mail: simon.straessle@physik.uzh.ch

R. Khasanov

Laboratory for Muon Spin Spectroscopy, Paul Scherrer Institut, 5232 Villigen PSI, Switzerland

T. Kondo · A. Kaminski

Ames Laboratory and Department of Physics and Astronomy, Iowa State University, Ames, IA 50011, USA

T. Kondo $\cdot$ T. Takeuchi

Department of Crystalline Materials Science, Nagoya University,

Nagoya 464-8603, Japan

D.O.G. Heron · S.L. Lee

School of Physics and Astronomy, University of St. Andrews, Fife, KY16 9SS, UK

T. Takeuchi

EcoTopia Science Institute, Nagoya University,

Nagoya 464-8603, Japan
Keywords Muon-spin rotation experiments - Cuprates · Suppressed superfluid density · Superconducting gap · Pseudogap

\section{Introduction}

An important open question in cuprate physics is the relevance of the pseudogap phenomenon for the occurrence of high-temperature superconductivity. The generic phase diagram of cuprate superconductors is determined by an antiferromagnetic, a pseudogap, and a superconducting phase, which are characterized by their respective transition temperatures $T_{N}, T^{*}$, and $T_{c}$. Below $T^{*}$ the pseudogap state emerges and the density of states at the Fermi level is decreased. Below $T_{c}$ the superconducting state appears. In the case that the pseudogap is a precursor phenomenon of superconductivity, long-range phase coherence will be established only below $T_{c}$ and all electronic states, including those affected by the occurrence of the pseudogap, begin to form the superconducting condensate. In other words, all electronic states over the whole Fermi surface, except for those at the nodes, contribute to superconductivity. However, a second possible scenario is that the superconducting condensate is formed only by those states which are not influenced by the opening of the pseudogap.

Various experiments support the latter scenario [1-6]. Angle resolved photoemission spectroscopy (ARPES) [3, 4] reveals that the energy gap which opens at $T_{c}$ exhibits point nodes along the diagonal of the $\mathrm{Cu}-\mathrm{O}$ bond, consistent with dominant $d$-wave superconductivity. Furthermore, it was shown that the pseudogap appears below $T^{*}$ and develops gradually to its maximum value towards the anti-nodal region of the Fermi arc, whereas it vanishes in a region near 
the nodes. In recent Raman-scattering experiments, an almost temperature independent anti-nodal gap in underdoped cuprates was found below $T^{*}$ [5]. In contrast to that, the energy gap in the near-nodal region opens below $T_{c}$ and follows a well-defined BCS temperature dependence [4]. In addition, the near-nodal gap scales with $T_{c}$ [4], whereas the anti-nodal gap size increases in general when lowering the doping in the underdoped regime [5]. These experimental observations suggest that the pseudogap and the superconducting state are coexisting phenomena, which may arise from different parts of the Fermi surface.

Muon-spin rotation $(\mu \mathrm{SR})$ experiments allow to deduce the magnetic penetration depth $\lambda$, which is related to the superfluid density as $\lambda^{-2} \propto \rho_{s} \propto n_{s} / m^{*}$, where $m^{*}$ and $n_{s}$ denote the supercarrier mass and density, respectively. This relation is valid within the London model $(\lambda \gg \xi)$. The temperature dependence of $\lambda$ is determined by the amplitude of the superconducting energy gap and its temperature and angular dependence. It is not possible to reconstruct the amplitude and the exact angular dependence directly from $\mu \mathrm{SR}$ data. However, by assuming $d$-wave superconductivity, $\lambda(T)$ can be reconstructed. This reconstruction yields a gap value. ARPES measurements provide the amplitude and the angular dependence of the energy gap. By comparing bulk-sensitive $\mu$ SR and surface-sensitive ARPES [3] experiments, different gap scenarios were tested. We find consistency between data of these two methods obtained for optimally doped $(\mathrm{BiPb})_{2}(\mathrm{SrLa})_{2} \mathrm{CuO}_{6+\delta}$ by assuming that superconductivity is caused only by states from the near-nodal part of the Fermi surface, i.e. that the anti-nodal states affected by the pseudogap do not participate in the superconducting condensate. The main results of this contribution are published in [7].

\section{Experimental Details}

The $\mu \mathrm{SR}$ experiments were conducted on $\mathrm{Pb}$ - and $\mathrm{Sr}$-doped single-crystalline $(\mathrm{BiPb})_{2}(\mathrm{SrLa})_{2} \mathrm{CuO}_{6+\delta}$ at optimum doping (OP Bi2201) with $T_{c}=35 \mathrm{~K}$ and a transition width $\sim 3 \mathrm{~K}$. The sample preparation is described elsewhere $[8,9]$. Exchanging Bi partially for $\mathrm{Pb}$ does not change $T_{c}$, but removes partially the buckling of the insulating $\mathrm{BiO}$ layer, which is relevant for ARPES studies [3]. The doping level is controlled by the relative amount of $\mathrm{Sr}$ and La.

OP Bi2201 is an ideal compound to investigate the connection between the superconducting and the pseudogap phase by means of $\mu \mathrm{SR}$ for the following reasons. First, recent ARPES studies provide detailed information on the amplitude and the angular dependence of the energy gap of OP Bi2201 [3]. Furthermore, a large difference between the two involved energy scales is advantageous, and the pseudogap size in OP Bi2201 is roughly three times larger than the superconducting one.
The transverse-field $\mu \mathrm{SR}$ experiments were carried out at the Paul Scherrer Institute (Villigen, Switzerland). Technical details of this method can be found in [10]. Two OP Bi2201 crystals with an approximate size of $4 \times 2 \times 0.1 \mathrm{~mm}^{3}$ were field-cooled to $1.6 \mathrm{~K}$ in fields ranging from 5 to $640 \mathrm{mT}$. The field was applied along the $c$ axis of the crystals, thus probing the decay of the magnetic field within the $a b$ plane $\left(\lambda_{a b}\right)$. The $\mu$ SR time spectra were analyzed by a twocomponent Gaussian approximation [7, 11, 12]:

$P(t)=\sum_{i=1}^{2} A_{i} \exp \left(-\sigma_{i}^{2} t^{2} / 2\right) \cos \left(\gamma_{\mu} B_{i} t+\phi\right)$,

from which the internal magnetic field distribution $P(B)$ of the superconductor in the mixed state was obtained by Fourier transformation:

$P(B)=\gamma_{\mu} \sum_{i=1}^{2} \frac{A_{i}}{\sigma_{i}} \exp \left(-\frac{\gamma_{\mu}^{2}\left(B-B_{i}\right)^{2}}{2 \sigma_{i}^{2}}\right)$.

$A_{i}, \sigma_{i}$, and $B_{i}$ denote the asymmetry, the relaxation rate, and the mean field of the $i$ th component, $\gamma_{\mu}=2 \pi \times$ $135.5342 \mathrm{MHz} / \mathrm{T}$ is the muon gyromagnetic ratio, and $\phi$ is the initial phase of the muon-spin ensemble.

$\lambda_{a b}$ was derived from $P(B)$ within the second-moment model [7, 11, 12]:

$\sigma_{\mathrm{tot}}^{2}=\sum_{i=1}^{2} \frac{A_{i}}{A_{1}+A_{2}}\left[\sigma_{i}^{2}+\gamma_{\mu}^{2}\left[B_{i}-\langle B\rangle\right]^{2}\right]$,

where $\lambda_{a b}^{-4} \propto \sigma_{\mathrm{tot}}^{2}-\sigma_{\mathrm{nm}}^{2}=\sigma_{\mathrm{sc}}^{2} . \sigma_{\mathrm{nm}}$ is the additional relaxation rate due to the nuclear moments, $\sigma_{\mathrm{sc}}$ is the superconducting state contribution, and $\langle B\rangle$ is the first moment of $P(B)$.

\section{Results and Discussion}

The field dependence of the second moment of $P(B)$ measured at $1.6 \mathrm{~K}$ is depicted in Fig. 1. The solid black line was calculated using the model of Brandt [13], assuming an isotropic $s$-wave superconductor with $\lambda=360 \mathrm{~nm}$ and $\xi \simeq 2.6 \mathrm{~nm}$. The coherence length $\xi$ was estimated from the second critical field $\mu_{0} H_{c 2}(0) \simeq 50 \mathrm{~T}$ [14]. In the case that the superconducting gap possesses nodes, a field dependent correction to the superfluid density $\rho_{s}$ due to its nonlinear and nonlocal response to the applied field has to be taken into account [15]. The nonlinear response of a $d$-wave superconductor is given by $[16,17]$

$\frac{\rho_{s}(H)}{\rho_{s}(H=0)}=\frac{\sigma_{\mathrm{sc}}(H)}{\sigma_{\mathrm{sc}}(H=0)}=1-K \sqrt{H}$. 




Fig. 1 (Color online) Magnetic field dependence of the in-plane $\sigma_{\mathrm{sc}} \propto \lambda_{a b}^{-2}$ of $(\mathrm{BiPb})_{2}(\mathrm{SrLa})_{2} \mathrm{CuO}_{6+\delta}$ at $1.6 \mathrm{~K}$. The solid black line results from numerical calculations using the model of Brandt [13], assuming an isotropic $s$-wave gap and $\lambda=360 \mathrm{~nm}$ and $\xi=2.6 \mathrm{~nm}$. For a $d$-wave superconductor the expected field dependence of $\sigma_{\mathrm{sc}}$ is represented by the solid red line, which includes a correction due to the nonlinear response of the in-plane superfluid density (see text). Examples of the asymmetric local magnetic field distribution $P(B)$ are plotted for 40 and $640 \mathrm{mT}$

The parameter $K$ measures the strength of the nonlinear response. Since (4) is valid for $H \gg H_{c 1}$, only fields exceeding $40 \mathrm{mT}$ were considered in the fit, which is represented by the red line in Fig. 1. The skewness parameter is derived from the second and the third moment of $P(B)$ according to $\alpha_{s}=\left\langle\Delta B^{3}\right\rangle^{\frac{1}{3}}\left\langle\Delta B^{2}\right\rangle^{-\frac{1}{2}}[18]\left(\left\langle\Delta B^{n}\right\rangle\right.$ is the $n$th moment of $P(B)) . \alpha_{s}(1.6 \mathrm{~K})$ was found to be field independent within error. Moreover, $\alpha_{s}=0.84(2)$ is smaller than the expected value of 1.2 for a triangular vortex lattice and $\kappa \gg 1$ [7]. The field independence of $\alpha_{s}$ ensures that no symmetry change of the vortex lattice takes place in the investigated field range. The magnetic field dependence of the in-plane $\sigma_{\mathrm{sc}} \propto \lambda_{a b}^{-2}$ shows consistency with a leading $d$-wave gap (see Fig. 1).

The temperature dependence of the second moment of $P(B)$ for a field of $40 \mathrm{mT}$ applied along the $c$-axis was studied (see Fig. 2(a)). Note that, below $20 \mathrm{~K}, \sigma_{\mathrm{sc}}(T)$ shows a linear behavior, consistent with the previous finding that there exist nodes in the gap of OP Bi2201.

The temperature dependence of $\lambda$ can be calculated using [12]

$$
\begin{aligned}
\frac{\lambda_{i}^{-2}(T)}{\lambda_{i}^{-2}(0)}= & +\frac{1}{\pi} \int_{0}^{2 \pi} \int_{\Delta(T, \varphi)}^{\infty} w(\varphi) \\
& \times\left(\frac{\partial f}{\partial E}\right) \frac{E d E d \varphi}{\sqrt{E^{2}-\Delta(T, \varphi)^{2}}},
\end{aligned}
$$

with

$\Delta(T, \varphi)=\Delta^{0} \times s\left(T / T_{c}\right) \times g(\varphi)$.

$i$ refers to the crystallographic axis $(i=a, b, c)$. With the quasi-particle weight function $w(\varphi)$ a non-uniform quasiparticle weight around the Fermi surface can be introduced (for a uniform quasi-particle weight: $w(\varphi)=1,0 \leq \varphi \leq$ $2 \pi) . f=\left[\exp \left(E / k_{B} T\right)+1\right]^{-1}$ denotes the Fermi distribution function, and $\lambda_{i}(0)$ and $\Delta^{0}$ are the zero-temperature values of the magnetic penetration depth and the superconducting gap, respectively. In $(6), g(\varphi)$ describes the angular dependence of the gap, whereas $s\left(T / T_{c}\right)$ describes its temperature dependence.

First, $\sigma_{\mathrm{sc}}(T)$ was analyzed assuming $d$-wave symmetry of the superconducting gap $[g(\varphi)=\cos (2 \varphi)]$ and a uniform quasi-particle weight around the Fermi surface $[w(\varphi)=1]$. For the temperature dependence of the gap, $s\left(T / T_{c}\right)=$ $\tanh \left(1.82\left[1.018\left(T_{c} / T-1\right)^{0.51}\right]\right)[19]$ was taken. To use the weak-coupling BCS formula for $s\left(T / T_{c}\right)$ is justified by direct gap measurements by means of ARPES [4] and tunneling spectroscopy [20]. By fitting the $\sigma_{\mathrm{sc}}(T)$ using (5), $\Delta^{0}=9.7(1) \mathrm{meV}$ was found $\left[2 \Delta^{0} / k_{B} T_{c}=6.43\right]$. The amplitude and the angular dependence of the superconducting gap used in this approach are illustrated by the black line in Fig. 2(b). Obviously, $\lambda(T)$ is well described by this analysis, see black line in Fig. 2(a), but the gap behavior seems to be inconsistent with the ARPES measurements shown in Fig. 2(b).

In a further step, we analyzed our $\mu$ SR data by directly introducing the amplitude and the angular dependence of the energy gap from ARPES measurements (see Fig. 2(b)) on a similar sample of OP Bi2201 [3]. Three different approaches were evaluated to reproduce the in-plane $\sigma_{\mathrm{sc}}(T)$ (see Fig. 2(a)) using the ARPES data (see Fig. 2(b)).

(a) In the first approach, $\Delta(\varphi)$ in (5) is given by the dashed green line in Fig. 2(b). Here, a uniform quasi-particle weight around the Fermi surface is assumed, i.e. $w(\varphi)=1$. The green line assumes a $d$-wave gap. The gap amplitude $\Delta^{0}$ is determined by fitting a $d$-wave gap expression $[g(\varphi)=$ $\cos (2 \varphi)]$ considering only those ARPES data points of the energy gap's angular dependence, which are not affected by the pseudogap phenomenon [3]. Thus, $\Delta(T, \varphi)=15 \mathrm{meV} \times$ $s\left(T / T_{c}\right) \times \cos (2 \varphi)\left[2 \Delta^{0} / k_{B} T_{c}=9.95\right]$. For the temperature dependence of the gap, $s\left(T / T_{c}\right)=\tanh \left(1.82\left[1.018\left(T_{c} / T-\right.\right.\right.$ $1)^{0.51}$ ]) [19] was taken. $\sigma_{\mathrm{sc}}(T)$ calculated within this approach is shown as a dashed green line in Fig. 2(a). Obviously, the dashed green line cannot describe the $\mu$ SR data.

(b) In the second approach, $\Delta(\varphi)$ in (5) is given by the solid blue line in Fig. 2(b). $w(\varphi)=1$ was assumed. The blue line is an analytical function consisting of two parts, 


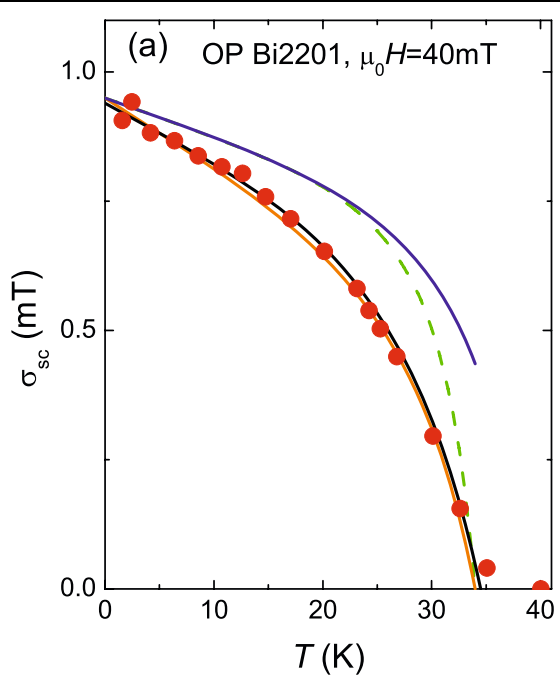

Fig. 2 (Color online) a Shows the temperature dependence of the in-plane $\sigma_{\mathrm{sc}} \propto \lambda_{a b}^{-2}$ of $(\mathrm{BiPb})_{2}(\mathrm{SrLa})_{2} \mathrm{CuO}_{6+\delta}$ for a field of $40 \mathrm{mT}$ applied along the $c$-axis. The lines are obtained using (5) and different amplitude $\Delta^{0}$ and angular dependence $g(\varphi)$ values of the energy gap $[g(\varphi)=\cos (2 \varphi)$ with a uniform quasi-particle weight around the Fermi surface with $\Delta^{0}=9.7(1) \mathrm{meV}$ (solid black) and $\Delta^{0}=15 \mathrm{meV}$ (dashed green), $g(\varphi)=\cos (2 \varphi)$ with partial suppression of the superfluid density (solid orange), and $g(\varphi)=\cos (2 \varphi)$

one describing the near-nodal and the other the near antinodal region. For the near-nodal part, $\Delta^{0}=15 \mathrm{meV}, g(\varphi)=$ $\cos (2 \varphi)$, and $s\left(T / T_{c}\right)=\tanh \left(1.82\left[1.018\left(T_{c} / T-1\right)^{0.51}\right]\right)$ [19] was taken. The near anti-nodal part was assumed to be temperature independent, as suggested by Ramanscattering experiments [5]. Furthermore, $\Delta^{0}=36 \mathrm{meV}$ and $g(\varphi)=\cos (3.4 \varphi)$ were taken. The $\sigma_{\mathrm{sc}}(T)$ calculated within this approach is shown as a solid blue line in Fig. 2(a). It is evident that also this approach cannot describe the $\mu \mathrm{SR}$ data.

(c) The third approach is the same as approach (a), except that here the superfluid density is assumed to be suppressed on a part of the Fermi surface, and is illustrated by the solid orange line in Fig. 2(b). Technically, this suppression can be realized by adjusting $w(\varphi)$ according to:

$w(\varphi)= \begin{cases}1 & \text { if }\left(n \cdot 45^{\circ}-25^{\circ}\right) \leq \varphi \leq n \cdot 45^{\circ}, \\ 0 & \text { else }\end{cases}$

Here, $n$ is an integer ranging from 1 to $8 . \sigma_{\mathrm{sc}}(T)$ calculated within this approach describes the $\mu$ SR data rather well and is shown as a solid orange line in Fig. 2(a).

Full consistency between bulk-sensitive $\mu$ SR and surface-sensitive ARPES measurements is obtained in the last approach (c), where a partial suppression of the quasiparticle weight was assumed. These findings therefore suggest that the pseudogap and the superconducting phase are dominated by different parts of the Fermi surface.

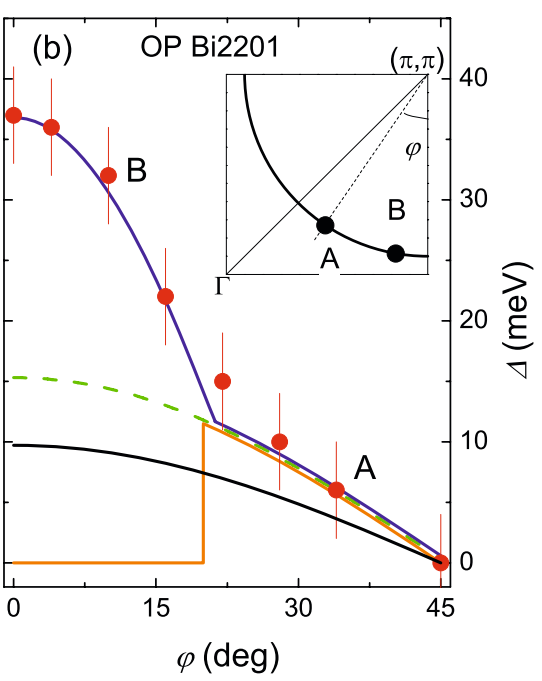

in the nodal and $g(\varphi)=\cos (3.4 \varphi)$ in the anti-nodal region (solid blue), see text for more details]. b Shows the amplitude and the angular dependence of the energy gap of OP Bi2201 as obtained from ARPES [3], and illustrates the different approaches used to analyze $\sigma_{\mathrm{sc}}(T)$ in the appropriate color. The inset in $\mathbf{b}$ shows schematically a part of the Fermi surface. The points A and $\mathrm{B}$ are in the near nodal and the near anti-nodal region, respectively

Note that both, the fitting procedure assuming a superconducting $d$-wave gap with a uniform quasi-particle weight over the whole Fermi surface with $\Delta^{0}=9.7 \mathrm{meV}$ (black line in Fig. 2(a)) and also the calculation using ARPES results assuming a superconducting $d$-wave gap with a partial suppression of the quasi-particles on the Fermi surface with $\Delta^{0}=15 \mathrm{meV}$ (orange line in Fig. 2(a)), describe our $\mu \mathrm{SR}$ data well.

\section{Conclusions}

From the field dependence of the $\mu$ SR data it was found that the in-plane superfluid density shows a behavior which excludes isotropic $s$-wave superconductivity for $(\mathrm{BiPb})_{2}$ $(\mathrm{SrLa})_{2} \mathrm{CuO}_{6+\delta}$ but shows consistency with superconductivity with a dominant $d$-wave order parameter. The comparison of the measured temperature dependence of $\lambda$ with calculation using recent ARPES measurements on a similar sample of the same compound suggests that the states affected by the pseudogap do not participate in the superfluid condensate. To further clarify this point, more experimental work is required.

Acknowledgements This work was performed at the Swiss Muon Source $(\mathrm{S} \mu \mathrm{S})$, Paul Scherrer Institute (Villigen, Switzerland). The authors are grateful to Y.J. Uemura and R. Prozorov for stimulating discussions. The support of the K. Alex Müller Foundation, the Swiss National Science Foundation, and the European CoMePhS Project 
is acknowledged. Work at the Ames Laboratory was supported by the Department of Energy-Basic Energy Sciences under Contract No. DE-AC02-07CH11358.

\section{References}

1. Le Tacon, M., Sacuto, A., Georges, A., Kotliar, G., Gallais, Y., Colson, D., Forget, A.: Nat. Phys. 2, 537 (2006)

2. Tanaka, K., Lee, W.S., Lu, D.H., Fujimori, A., Fujii, T., Risdiana, Terasaki, I., Scalapino, D.J., Devereaux, T.P., Hussain, Z., Shen, Z.-X.: Science 314, 1910 (2006)

3. Kondo, T., Takeuchi, T., Kaminski, A., Tsuda, S., Shin, S.: Phys. Rev. Lett. 98, 267004 (2007)

4. Lee, W.S., Vishik, I.M., Tanaka, K., Lu, D.H., Sasagawa, T., Nagaosa, N., Devereaux, T.P., Hussain, Z., Shen, Z.-X.: Nature (London) 450, 81 (2007)

5. Guyard, W., Le Tacon, M., Cazayous, M., Sacuto, A., Georges, A., Colson, D., Forget, A.: Phys. Rev. B 77, 024524 (2008)

6. Hanaguri, T., Kohsaka, Y., Davis, J.C., Lupien, C., Yamada, I., Azuma, M., Takano, M., Ohishi, K., Ono, M., Takagi, H.: Nat. Phys. 3, 865 (2007)

7. Khasanov, R., Kondo, T., Strässle, S., Heron, D.O.G., Kaminski, A., Keller, H., Lee, S.L., Takeuchi, T.: Phys. Rev. Lett. (2008, accepted). arXiv:0806.1907

8. Kondo, T., Takeuchi, T., Yokoya, T., Tsuda, S., Shin, S., Mizutani, U.: J. Electron Spectrosc. Relat. Phenom. 137-140, 663 (2004)
9. Kondo, T., Takeuchi, T., Mizutani, U., Yokoya, T., Tsuda, S., Shin, S.: Phys. Rev. B 72, 024533 (2005)

10. Sonier, J.E., Brewer, J.H., Kiefl, R.F.: Rev. Mod. Phys. 72, 769 (2000)

11. Khasanov, R., Landau, I.L., Baines, C., La Mattina, F., Maisuradze, A., Togano, K., Keller, H.: Phys. Rev. B 73, 214528 (2006)

12. Khasanov, R., Shengelaya, A., Maisuradze, A., La Mattina, F., Bussmann-Holder, A., Keller, H., Müller, K.A.: Phys. Rev. Lett. 98, 057007 (2007)

13. Brandt, E.H.: Phys. Rev. B 68, 054506 (2003)

14. Wang, Y., Ono, S., Onose, Y., Gu, G., Ando, Y., Tokura, Y., Uchida, S., Ong, N.P.: Science 299, 86 (2003)

15. Amin, M.H.S., Franz, M., Affleck, I.: Phys. Rev. Lett. 84, 5864 (2000)

16. Vekhter, I., Carbotte, J.P., Nicol, E.J.: Phys. Rev. B 59, 1417 (1999)

17. Won, H., Maki, K.: Europhys. Lett. 54, 248 (2001)

18. Lee, S.L., Zimmermann, P., Keller, H., Warden, M., Savić, I.M., Schauwecker, R., Zech, D., Cubitt, R., Forgan, E.M., Kes, P.H., Li, T.W., Menovsky, A.A., Tarnawski, Z.: Phys. Rev. Lett. 71, 3862 (1993)

19. Carrington, A., Manzano, F.: Physica C 385, 205 (2003)

20. Ichimura, K., Nomura, K., Minami, F., Takekawa, S.: J. Phys.: Condens. Matter 2, 9961 (1990) 